\title{
Minimally invasive optical biopsy for oximetry
}

\author{
Marieke A. van der Putten ${ }^{\mathrm{a}}$, James M. Brewer ${ }^{\mathrm{b}}$, and Andrew R. Harvey ${ }^{\mathrm{a}}$

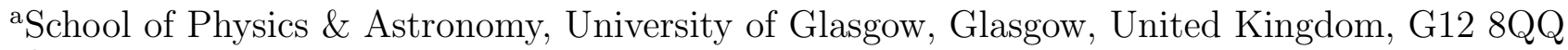 \\ ${ }^{b}$ Institute for Infection, Immunity \& Inflammation, University of Glasgow, Glasgow, United \\ Kingdom, G12 8QQ
}

\begin{abstract}
The study of localised oxygen saturation in blood vessels can shed light on the etiology and progression of many diseases with which hypoxia is associated. For example, hypoxia in the tendon has been linked to early stages of rheumatoid arthritis, an auto-immune inflammatory disease. Vascular oximetry of deep tissue presents significant challenges as vessels are not optically accessible. In this paper, we present a novel multispectral imaging technique for vascular oximetry, and recent developments made towards its adaptation for minimally invasive imaging. We present proof-of-concept of the system and illumination scheme as well as the analysis technique. We present results of a validation study performed in vivo on mice with acutely inflamed tendons. Adaptation of the technique for minimally invasive microendoscopy is also presented, along with preliminary results of minimally invasive ex vivo vascular oximetry.
\end{abstract}

Keywords: Multispectral imaging, oximetry, microendoscopy, vasculature

\section{INTRODUCTION}

A healthy supply of oxygen is of great importance for the proper function of cells and tissues. Oxygen is supplied throughout the body by the vasculature, with oxygen diffusing directly into tissue from networks of capillaries known as the microvasculature. Vascular oximetry is the measurement of oxygen saturation in the blood vessels of the body, made possible by the distinct absorption spectra of oxygenated and deoxygenated haemoglobin. Hypoxia is an inadequate supply of oxygen in tissue, and its detection can be indicative of a range of diseases, for example a dysfunctional oxygen network in the retina is a symptom of diabetic retinopathy and glaucoma. ${ }^{1,2}$ Hypoxia has also been linked to auto-immune inflammatory disease such as multiple sclerosis $(\mathrm{MS})^{3}$ and rheumatoid arthritis (RA). ${ }^{4}$

To date, imaging and analysis techniques for vascular oximetry have predominantly been developed for application to optically accessible vascular beds such as in the eye. ${ }^{5-7}$ Localised vascular oximetry in deeper tissue remains challenging as it not optically accessible, requiring invasive procedure for access. In this work we present progress towards a minimally invasive oximetric microscope, capable of accessing vasculature within deep tissue and measuring absolute oxygen saturation $\left(\mathrm{SO}_{2}\right)$ by means of multispectral imaging and employing a triplet-lens microendoscope. The complete configuration of this imaging system is described in section 2.1. Although suitable for a range of applications, the validations of the technique presented here focus on oximetry of the vasculature in murine tendon tissue; both ex vivo and in vivo. Tendon tissue affected by RA is associated with painful swelling and inflammation. Whilst hypoxia has been linked to early stages of the disease, the relationship between oxygen saturation and disease progression is not thoroughly understood. The ability to perform longitudinal oximetry studies with models for inflammation of RA would be highly desirable for providing insight into the link between hypoxia and RA, and is the motivating factor for this chosen application.

Microendoscopic imaging has become an active field of research over the past several years, with many studies specifically focused on minimally invasive applications of fluorescence microscopy. ${ }^{8-10}$ Recently, some progress has been made towards implementing GRIN lens technologies for the purposes of monitoring oxygen saturation. ${ }^{11}$ However in that paper, only superficial sublingual and labial blood vessels were studied, and

Further author information: (Send correspondence to A.R.H)

A.R.H.: E-mail: andy.harvey@glasgow.ac.uk, Telephone: +44 (0)141 3308606

Endoscopic Microscopy XII, edited by Guillermo J. Tearney, Thomas D. Wang, Proc. of SPIE Vol. 10040, 1004009 - @ 2017 SPIE · CCC code: 1605-7422/17/\$18 · doi: 10.1117/12.2253645 
images were acquired at only two wavelengths. Thus, only measurements of optical density ratios (ODRs) of the vasculature were produced. ODRs are linearly proportional to oxygen saturation, and hence can be useful as a proxy for oximetry. However, it is not possible to determine absolute values for oxygen saturation with ODRs, without prior calibration. This is not practical for in vivo applications where the optical environment can change locally. To the best of our knowledge, we present the first demonstration of absolute vascular oximetry of $e x$ vivo deep tissue using microendoscopic probes. We describe the basic multispectral imaging system and analysis technique as well as ex vivo and in vivo validation. We then present a summary of how this system has been adapted for minimally invasive oximetry, including preliminary results of an experiment imaging vasculature in ex vivo tendon tissue. Work is now ongoing to apply the techniques and methods presented here to longitudinal oximetry studies of tendons in vivo which are affected by models of RA.

\section{METHODS}

\subsection{Multispectral Imaging}

The initial oximetric microscope developed for preliminary proof-of-concept experiments was based on a standard sequential multispectral imaging system, adapted with a custom annular illumination scheme to provide diffuse back-illumination of capillaries. In sequential multispectral imaging, images are recorded at each waveband in quick succession. Spectral discrimination was provided by a white-light supercontinuum source (Fianium SC400) coupled to an acousto-optic tunable filter (AOTF, Gooch and Housego) with a nominal $2 \mathrm{~nm}$ bandwidth. A 12 bit CCD camera (Hamamatsu C4742-95-12ER)was used as the detector. A 40X reverse-cassegrain type reflective objective was employed in order to minimise chromatic aberrations. Further, the use of a reflective objective and its inherent obscuration (due to the placement of the primary mirror) allowed for annular illumination to be generated around the field of view. The advantages of using annular illumination for vascular oximetry are explained in section 2.3. As the AOTF output is spatially coherent, an oscillating polymer diffuser was used to de-speckle the image.

Six wavelengths were chosen for imaging of the microvasculature; $410 \mathrm{~nm}, 420 \mathrm{~nm}, 430 \mathrm{~nm}, 435 \mathrm{~nm}, 440 \mathrm{~nm}$ and $450 \mathrm{~nm}$. Figure 1 shows the molar extinction spectra of deoxygenated and oxygenated haemoglobin respectively. Absorption is higher in this visible blue region than for longer wavelengths such as the green or red commonly used for retinal oximetry. ${ }^{12,13}$ Use of highly absorbing blue wavelengths is necessary for sufficient contrast of the small microvasculature against the white tendon background. At longer wavelengths with lower extinction coefficients as typically employed in retinal oximetry where major vessels are approximately $100 \mu \mathrm{m}$, smaller vessels become too transparent for accurate oximetry.

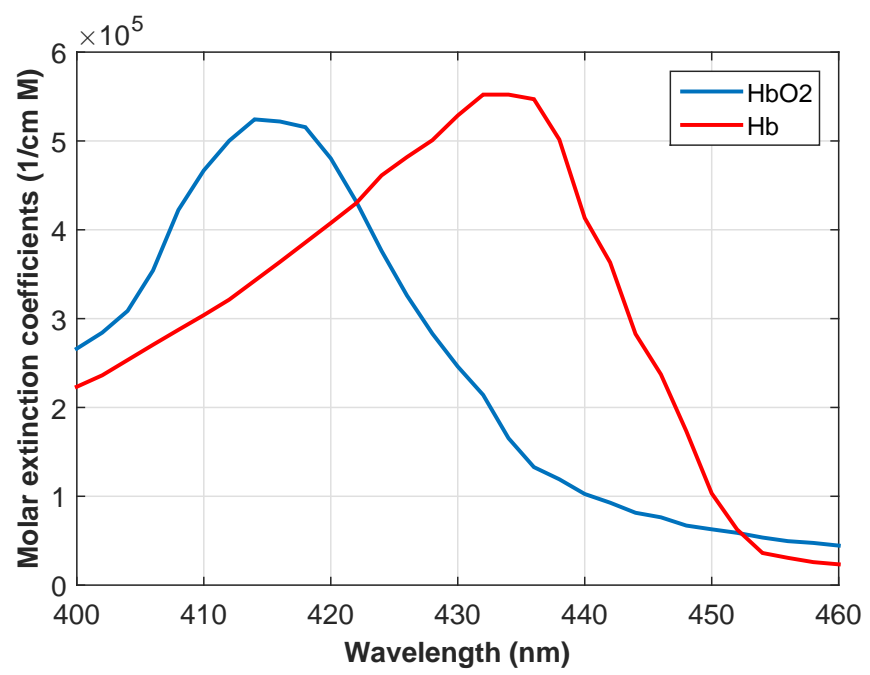

Figure 1. Molar extinction coefficient spectra for oxygenated $\left(\mathrm{HbO}_{2}\right)$ and deoxygenated haemoglobin $(\mathrm{Hb})$ in the visible blue wavelength region. Data from Prahl (1999). ${ }^{14}$ 
With this initial multispectral imaging system, preliminary ex vivo and in vivo experiments were performed to validate sensitivity to measurement of $\mathrm{SO}_{2}$ values and detection of changes to oxygenation.

For the first ex vivo experiment, tendon tissue of a mouse hind leg was used. It is generally accepted that post mortem, blood vessels become depleted of oxygen, due to localised metabolism of the remaining oxygen by cells in the vicinity. Thus, ex vivo tendon tissue will be deoxygenated. However, superficial blood vessels will reoxygenate when exposed to air, due to diffusion of oxygen. To demonstrate a measured increase in oxygenation, a mouse hind leg was placed in a small chamber through which nitrogen was flowing; the skin was then removed, exposing superficial vasculature on the tendon. The nitrogen prevented the blood vessels from reoxygenating, and the vasculature was imaged. The nitrogen flow was then stopped, and the same vasculature was imaged five minutes later. The results of this experiment are presented in section 3 .

For the preliminary in vivo work, 12 adult female BALB/c mice (18-25 g, 6-8 weeks) were used. The aim of this experiment was to investigate whether hypoxia associated with inflammation could be measured using our imaging and analysis technique. All animal work was performed in accordance with UK Home Office guidelines. Six of the mice were inoculated with $\lambda$-carrageenan, by means of an injection to the left hind footpad with $25 \mu \mathrm{L}$ of $1 \%$ solution in phosphate buffered saline. Carrageenan is a polysaccharide which is known to induce an acute inflammatory response which peaks 5 hours after inoculation. ${ }^{15}$ For validation of successful inoculation, measurement of mouse footpad thickness was performed before and after inoculation with $\lambda$-carrageenan, 5 hours post-injection. Average footpad thickness increased from $1.6 \pm 0.1 \mathrm{~mm}$ to $3.0 \pm 0.3 \mathrm{~mm}$ in this time, indicating that acute inflammation had occurred. The remaining six mice were used as controls. For this initial work, it was required to surgically expose the tendon by removal of the skin. For future minimally invasive imaging employing microendoscopes, this surgery will not be necessary. The surgical intervention and subsequent imaging sessions were performed under anaesthesia. For delivery of anaesthesia, a solution of fentanyl-fluanisone (Hypnorm), midazolam (Hypnovel) and water (1:1:2 by volume) was injected intraperitonally to each mouse at a dose of $10 \mu \mathrm{l} / \mathrm{g}$. The results of this experiment are also described in section 3.

\subsection{Incorporation of Microendoscope for Minimally Invasive Imaging}

The microendoscope employed for minimally invasive oximetry was a custom GRIN-lens triplet probe (Grintech GmbH, GT-IRLS-035-50-20-50-NC-ST) as depicted in figure 2. The microendoscope had a total physical length of $9.8 \mathrm{~mm}$, with imaging and coupling lens NA's of 0.5 , and relay lens NA of 0.2 . The diameter of the lens is $350 \mu \mathrm{m}$. The effective field of view is related to the lens diameter and ratio of imaging and relay lens NA's, ${ }^{16}$ and is approximately $140 \mu \mathrm{m}$. The microendoscope has a working distance of $100 \mu \mathrm{m}$ in air, specified for a nominal operating wavelength of $450 \mathrm{~nm}$.

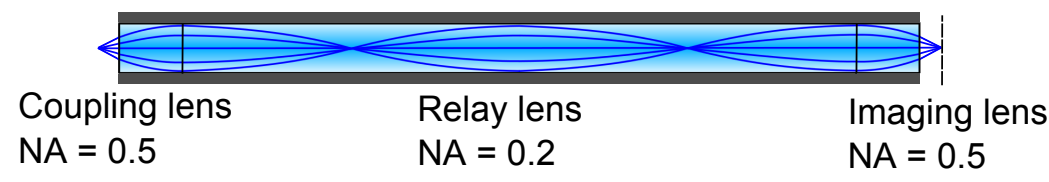

Figure 2. Illustration of triplet GRIN lens probe choice for minimally invasive vascular oximetry. The sinusoidal paths taken by incident rays are shown in blue.

To incorporate the microendoscope to the multispectral imaging system, the probe was placed beneath the reflective objective such that the object plane of the objective was coincident with the image plane of the microendoscope. In order to attain the annular illumination scheme described in section 2.1, it was necessary to employ ancillary optical fibres to deliver illumination about the field of view. A custom triplet optical fibre bundle was assembled for this purpose. The distal ends of the fibres were carefully affixed to the probe using UVcured epoxy, equally spaced around the metallic sheath which encompasses the microendoscope. Using ancillary 
illumination in this manner allows for more light to be delivered directly to the tissue - using the microendoscope itself for propagation of illumination results in loss of light as the transmission efficiency of these type of probes is less than $70 \% \cdot{ }^{16,17}$ The probe and fibres are held in position beneath the objective with a clamp (Thorlabs Micro V-Clamp) secured to a 3-axis translation stage. Additional UV-cured epoxy is used to ensure a tight connection between the probe and the clamp - without epoxy, there is a tendency for the probe to slip upwards if brought in contact with an object such as biological tissue. Figure 3 shows a schematic of the imaging system adapting for minimally invasive oximetry. Multispectral illumination is sequentially controlled by means of a Labview interface which switches the AOTF and triggers the CCD at each waveband. A complete multispectral dataset of 6 wavebands takes approximately 2 seconds to acquire, limited by the exposure time required at each waveband. Whilst snapshot multispectral techniques exist, ${ }^{12,18}$ sequential imaging is sufficient for a range of applications such as this, where movement of the sample is minimal.

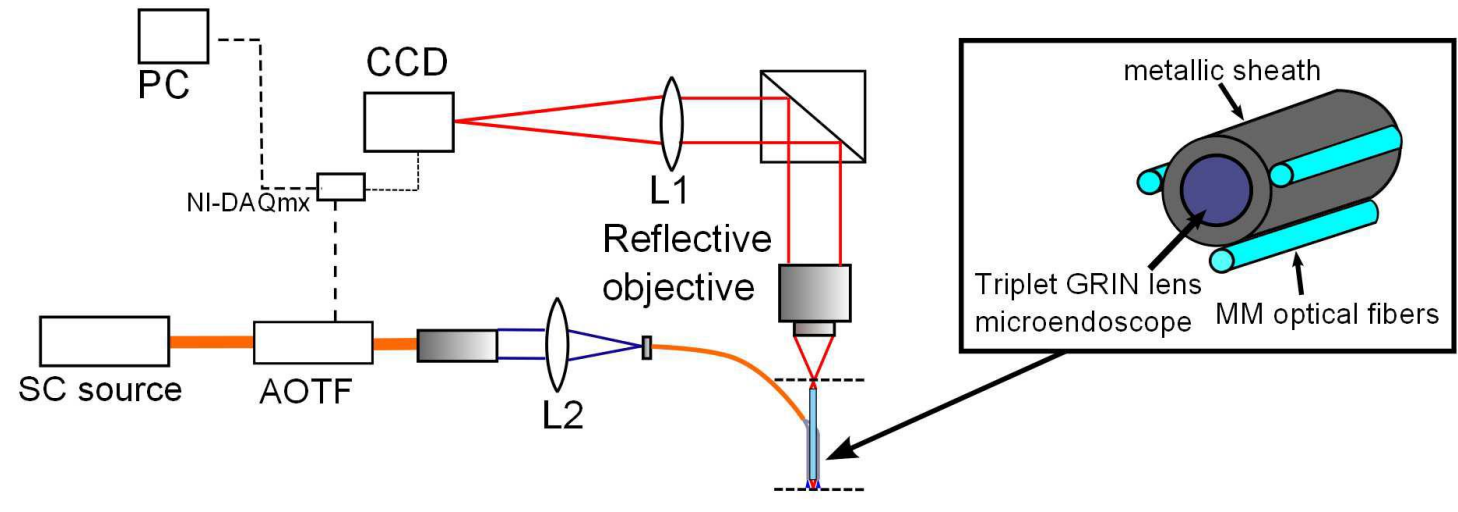

Figure 3. Multispectral imaging system with incorporation of microendoscopic probe and ancillary illumination. L1 = $200 \mathrm{~mm}$ tube lens; L2 = lens for fiber coupling; SC = supercontinuum; AOTF = acousto-optic tunable filter

\subsection{Oximetry Analysis}

For oximetry analysis of multispectral data, we employ a custom algorithm based on previous work by van der Putten et al., (2016) ${ }^{19}$ and by Smith et al., (2000). ${ }^{20}$ We derived a custom analytical model for the transmission of light through blood vessels at each waveband, described in equation 1.

$$
\begin{aligned}
T(\lambda)=s \cdot 10^{-}\left(C_{H b}(\lambda) \eta d\left[\left(\varepsilon_{H b O_{2}}(\lambda)-\varepsilon_{H b}(\lambda)\right) S O_{2}+\varepsilon_{H b}(\lambda)\right]+\right. \\
{\left.\left[\left(\mu_{H b O_{2}}^{\prime}(\lambda)-\mu_{H b}^{\prime}(\lambda)\right) S O_{2}+\mu_{H b}^{\prime}(\lambda)\right] \eta d\right)(1-K)+K }
\end{aligned}
$$

The above model takes into account both the extinction $\left(\varepsilon_{H b}\right.$ and $\left.\varepsilon_{H_{b} O_{2}}\right)$ and scattering coefficients $\left(\mu_{H b}^{\prime}\right.$ and $\mu_{\mathrm{HbO}_{2}}^{\prime}$ ) of oxygenated and deoxygenated haemoglobin respectively (from Prahl (1999) ${ }^{14}$ and Friebel et al., $(2009)^{21}$ ). We also incorporate a 'contrast reduction' factor, $K$ (where $0 \leq K \leq 1$ ) to account for loss of contrast (e.g. higher effective transmission) due to minor local fluctuations in overlaying tissue thickness, as well as loss of contrast due to the OTF of the imaging system. We also account for average haemoglobin concentration $C_{H b}$. Finally, $\eta$ is a parameter which accounts for the light path taken by detected photons - for vascular oximetry, detected light is expected to be predominantly composed of single-pass, double-pass and back-scattered light. ${ }^{22}$ For purely single-pass illumination $\eta=1$, whilst purely double-pass illumination $\eta$ would be 2 . As mentioned previously, we employ an annular illumination scheme, so we can make the assumption that only light which has diffused through adjacent tissue will pass through the blood vessels, resulting in single-pass illumination. As such, we set $\eta$ equal to 1 for this application, reducing the number of free variables. For determination of $\mathrm{SO}_{2}$, coordinates along blood vessels are selected using a semi-automatic vessel tracking algorithm, from which 
optical transmission at each waveband is measured by applying two fits perpendicularly across the vessel at each coordinate pair. Transmission $T$ is defined by $T=\frac{I}{I_{o}}$, where $I$ is the measured intensity of light in the centre of the vessel, and $I_{O}$ the measured intensity of light directly adjacent. A linear fit is applied to the perpendicular line profile to determine $I_{o}$, and a third-order polynomial fit is applied to the central vessel region for determination of $I$. A least-squares fit is then performed to optiimize the model with respect to the experimentally obtained "transmission profiles" generated along selected blood vessels at each wavelength, in order to produce values for parameters such as $\mathrm{SO}_{2}$ and $K$. This is performed using a trust-region reflective algorithm. For the blue wavelength regime employed for this analysis, simulations showed that the calculated values for $\mathrm{SO}_{2}$ are highly robust to noise (e.g. resulting from low SNR images). For additive gaussian noise of variance 0.005, all $\mathrm{SO}_{2}$ values (observed in $10 \%$ intervals from $0 \%$ to $100 \%$ ) deviated from the expected value by less than $6 \%$ in all cases.

\section{RESULTS}

\subsection{Proof-of-concept ex vivo and in vivo experiments}

The results of the initial ex vivo experiment are displayed in figure 4. For two deoxygenated ex vivo tendons, cessation of nitrogen flow in the enclosing chamber caused vasculature oxygenation to increase from an average of $34.7 \pm 8.5 \%$ to $92.6 \pm 4.2 \%$ in one case (mean \pm standard deviation, $\mathrm{p} \ll 0.01$ ), and $16.2 \pm 3.5 \%$ to $96.3 \pm 1.9 \%$ in the other $(\mathrm{p} \ll 0.01)$. Thus we initially demonstrated that changes in oxygen saturation are detectable using the illumination scheme and analysis technique, with physiologically plausible absolute values for $\mathrm{SO}_{2}$.

The in vivo experiment was performed to investigate whether it was possible to identify and measure hypoxia associated with acute inflammation. Acute inflammation and swelling is associated with auto-immune inflammatory disease such as RA, and the $\lambda$-carrageenan model for inflammation was chosen for this reason. The results are displayed in figure 5. As evident from the graph, there was a significant decrease in the measured $\mathrm{SO}_{2}$ of inflamed tendons when compared to the controls. Average $\mathrm{SO}_{2}$ for all control tendons was $94.8 \pm 7.0 \%$, with inflamed tendons averaging $84.0 \pm 13.5 \%(\mathrm{p}<0.01)$. Further, the decrease in $\mathrm{SO}_{2}$ of each inflamed tendon was statistically significant when compared to each control. We have thus demonstrated the first localised measurement of $\mathrm{SO}_{2}$ and hypoxia in vasculature affected by inflammation.

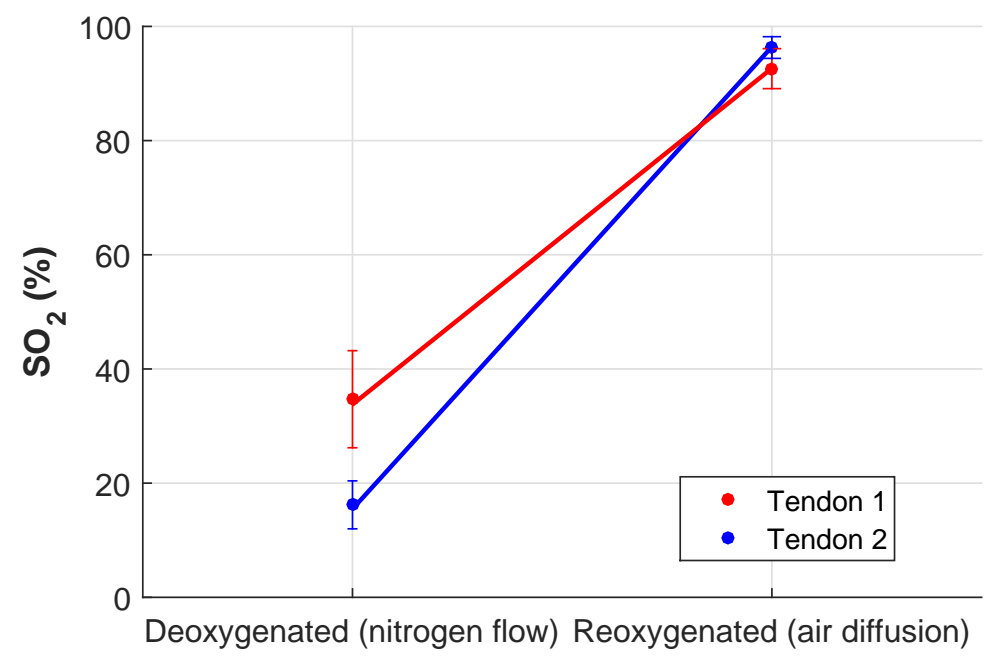

Figure 4. Results of ex vivo experiment demonstrating re-oxygenation of deoxygenated tendon vasculature upon cessation of nitrogen flow in chamber. Vessel becomes oxygenated due to exposure with oxygen. Red and blue plots represent two individual tendons 


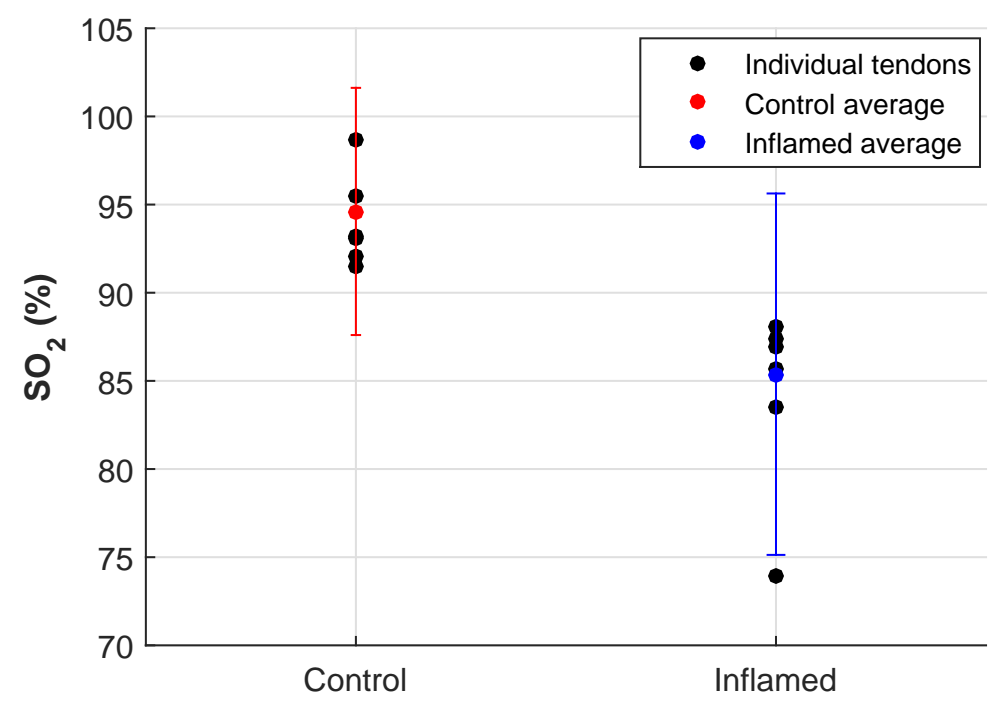

Figure 5. Results of in vivo proof-of-concept experiment demonstrating detection of hypoxia present in inflamed murine tendon

\subsection{Preliminary microendoscopy results}

Initial checks with the minimally invasive oximetric technique have been performed. Figure 6(a) shows a representative example of an image formed of a blood vessel through the microendoscope. The outer circular edge of the field of view is visible, as is inherent vignetting of the image towards this edge. The illumination was provided by the ancillary optical fibers surrounding the probe, which was scattered through tissue and into the field of view; there was no direct illumination provided through the microendoscope itself.

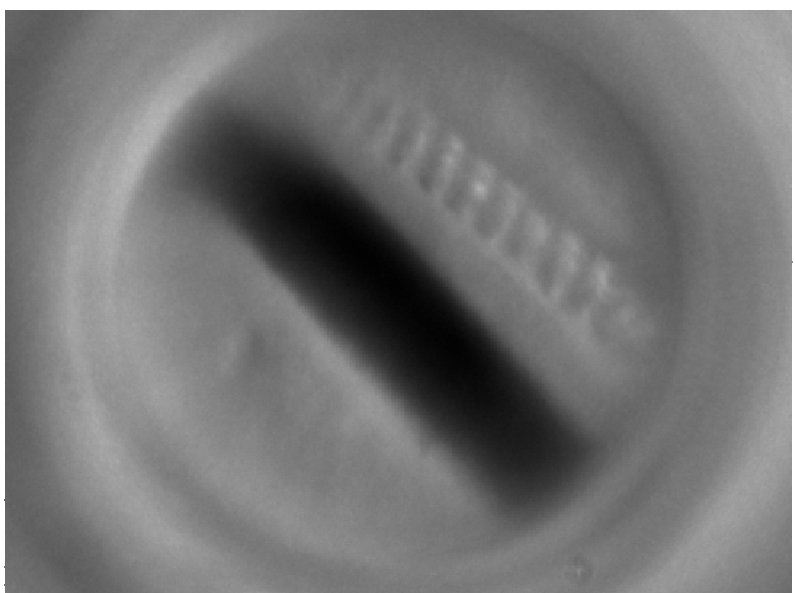

(a) Representative image of blood vessel

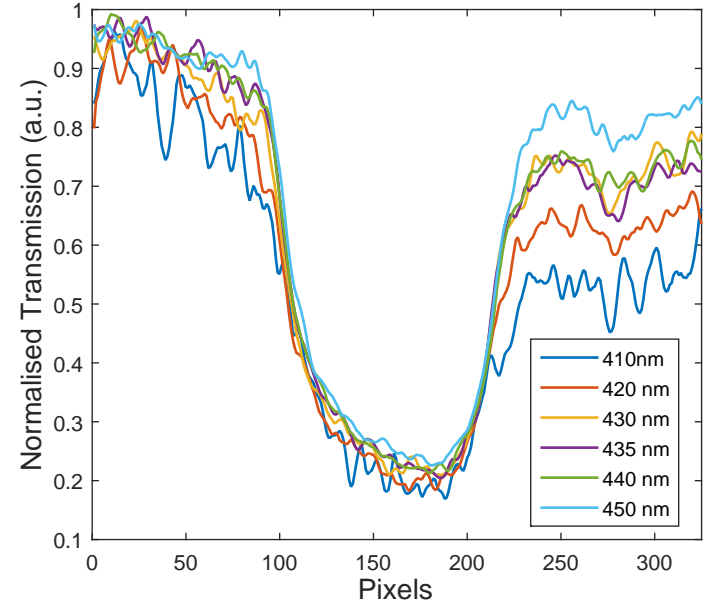

(b) Representative example of transverse line profiles across vessel.

Figure 6. Example of data from microendoscopic ex vivo multispectral imaging of tendon vasculature as imaged through probe and illuminated by diffuse light from ancillary fiber configuration

An experimentally obtained transverse line profile is shown in figure $6(\mathrm{~b})$, with profiles from each of the six 
wavebands shown. As expected, transmissions are lowest for $410 \mathrm{~nm}$ and $420 \mathrm{~nm}$, where extinction coefficients are highest for oxygenated haemoglobin.

\section{CONCLUSIONS AND FUTURE WORK}

The feasibility of the imaging system and oximetry analysis has been thoroughly demonstrated through means of preliminary proof-of-concept experiments ex vivo and in vivo. Further, the extension of the technique to minimally invasive imaging with a microendoscope and custom fibre-illuminated probe has been recently demonstrated. Current work is now focused on improving light delivery to the tissue for higher SNR, and pursuing a longitudinal study of microvasculature in the tendon, using a model for rheumatoid arthritis. With future results, it will be possible to investigate potential correlates between hypoxia and the stage of disease progression. This will hopefully shed light on the etiology and progression of the disease and pave the way for enhanced diagnostic capability, ultimately improving patient prognoses. In future it may also be desirable to combine snapshot multispectral techniques with microendoscopy, as mentioned previously ${ }^{12,18}$ allowing for real-time oximetric analysis of the vasculature with high temporal resolution. The challenge remains sufficient delivery of light into tissue for images with adequate SNR for accurate oximetry analysis.

\section{ACKNOWLEDGMENTS}

This work is funded by the University of Glasgow's 'Lord Kelvin Adam Smith' scholarship for interdisciplinary research.

\section{REFERENCES}

[1] Hammer, M., Vilser, W., Riemer, T., Mandecka, A., Schweitzer, D., Kühn, U., Dawczynski, J., Liemt, F., and Strobel, J., "Diabetic patients with retinopathy show increased retinal venous oxygen saturation," Graefe's Archive for Clinical and Experimental Ophthalmology 247(8), 1025-1030 (2009).

[2] Boeckaert, J., Vandewalle, E., and Stalmans, I., "Oximetry: recent insights into retinal vasopathies and glaucoma.," Bulletin de la Société belge d'ophtalmologie (319), 75-83 (2012).

[3] Davies, A. L., Desai, R. a., Bloomfield, P. S., McIntosh, P. R., Chapple, K. J., Linington, C., Fairless, R., Diem, R., Kasti, M., Murphy, M. P., and Smith, K. J., "Neurological deficits caused by tissue hypoxia in neuroinflammatory disease," Annals of Neurology 74(6), 815-825 (2013).

[4] Eltzschig, H. K. and Carmeliet, P., "Hypoxia and inflammation.," The New England journal of medicine 364(20), 1976-1977 (2011).

[5] Delori, F., "Noninvasive technique for oximetry of blood in retinal vessels," Applied Optics 27(6), 1113-1125 (1988).

[6] Beach, J., Schwenzer, K., Srinivas, S., Kim, D., and Tiedeman, J., "Oximetry of retinal vessels by dualwavelength imaging: calibration and influence of pigmentation," Journal of Applied Physiology 86(2), 748758 (1999).

[7] MacKenzie, L. E., Choudhary, T. R., McNaught, A. I., and Harvey, A. R., "In vivo oximetry of human bulbar conjunctival and episcleral microvasculature using snapshot multispectral imaging," Experimental Eye Research 149, 48-58 (2016).

[8] Barretto, R. P. J., Messerschmidt, B., and Schnitzer, M. J., "In vivo fluorescence imaging with highresolution microlenses.," Nature methods 6(7), 511-2 (2009).

[9] Saunter, C. and Semprini, S., "Micro-endoscope for in vivo widefield high spatial resolution fluorescent imaging," Biomedical Optics Express 3(6), 1274-1278 (2012).

[10] Yan, W., Peng, X., Lin, D., Wang, Q., Gao, J., Luo, T., Zhou, J., Ye, T., Qu, J., and Niu, H., "Fluorescence microendoscopy imaging based on GRIN lenses with one- and two-photon excitation modes," Frontiers of Optoelectronics 8(2), 177-182 (2015).

[11] Townsend, D., D'Aiuto, F., and Deanfield, J., "In Vivo Capillary Loop Hemoglobin Spectroscopy in Labial, Sublingual, and Periodontal Tissues," Microcirculation 22(6), 475-484 (2015). 
[12] Choudhary, T., Ball, D., Fernandez Ramos, J., McNaught, A., and Harvey, A., "Assessment of acute mild hypoxia on retinal oxygen saturation using snapshot retinal oximetry," Investigative ophthalmology $\& 3$ visual science 54(12), 7538-43 (2013).

[13] Beach, J., "Pathway to retinal oximetry.," Transl Vis Sci Technol 3(5) (2014).

[14] Prahl, S., "Optical Absorption of Hemoglobin, http://omlc.org/spectra/hemoglobin/," (1999).

[15] Morris, C. J., [Carrageenan-Induced Paw Edema in the Rat and Mouse], vol. 225 (2003).

[16] Li, X. and $\mathrm{Yu}, \mathrm{W} .$, "Deep tissue microscopic imaging of the kidney with a gradient-index lens system," Optics Communications 281, 1833-1840 (2008).

[17] Levene, M. J., Dombeck, D. a., Kasischke, K. a., Molloy, R. P., and Webb, W. W., "In vivo multiphoton microscopy of deep brain tissue.," Journal of neurophysiology 91(4), 1908-12 (2004).

[18] Gorman, A., Fletcher-Holmes, D. W., and Harvey, A. R., "Generalization of the Lyot filter and its application to snapshot spectral imaging.," Optics express 18(6), 5602-8 (2010).

[19] van der Putten, M. A., MacKenzie, L., Davies, A., Fernandez-Ramos, J., Desai, R., Smith, K., and Harvey, A., "A multispectral microscope for in vivo oximetry of rat dorsal spinal cord vasculature," Physiological Measurement https://doi.org/10.1088/1361-6579/aa5527 (2016).

[20] Smith, M. and Denninghoff, K., "Effect of multiple light paths on retinal vessel oximetry," Applied optics 39(7), 1183-93 (2000).

[21] Friebel, M., Helfmann, J., Netz, U., and Meinke, M., "Influence of oxygen saturation on the optical scattering properties of human red blood cells in the spectral range 250 to $2000 \mathrm{~nm}$," Journal of Biomedical Optics 14(3), 034001 (2009).

[22] Rodmell, P. I., Crowe, J. a., Gorman, A., Harvey, A. R., Muyo, G., Mordant, D. J., McNaught, A. I., and Morgan, S. P., "Light path-length distributions within the retina.," Journal of biomedical optics 19(3), 36008 (2014). 